\title{
Far UV-C lights and fiber optics induced and selective far UV-C treatment against COVID-19 for fatality-survival tradeoff
}

This paper was downloaded from TechRxiv (https://www.techrxiv.org).

\section{LICENSE}

CC BY 4.0

\section{SUBMISSION DATE / POSTED DATE}

$25-04-2020$ / 27-04-2020

\section{CITATION}

Haider, Imran; Ali, Asad; Arifeen, Tooba; Hassan, Abdus Sami (2020): Far UV-C lights and fiber optics induced and selective far UV-C treatment against COVID-19 for fatality-survival tradeoff. TechRxiv. Preprint. https://doi.org/10.36227/techrxiv.12195870.v1

$\mathrm{DOI}$ 


\title{
Far UV-C Lights and Fiber Optics Induced Selective Far UV-C treatment against COVID-19 for Fatality-Survival tradeoff
}

\author{
Imran Haider1, Asad Ali2, Tooba Arifeen ${ }^{3}$, and Abdus Sami Hassan 4 \\ Im.haider@gmail.com¹, asad.ali.arifeen@gmail.com², tooba.arifeen@gmail.com³ , a.sami.hassan@gmail.com 4
}

\begin{abstract}
The global pandemic of severe acute respiratory syndrome, COVID-19 has put the world to a standstill. Human mobility has turned into a threat which ultimately effects the livelihood of the homo sapiens. This work focuses on the usability of Ultraviolet (UV) on humans as an economical way of contesting against COVID-19. Specifically, far-UVC is presented as a promising candidate against COVID-19 since it can inactivate pathogens including viruses and bacteria without harming mammalian skin or eyes. Furthermore, the work also points out the applicability of far-UVC lights for public spaces and treatment of selective region in COVID-19 patients by using fiberoptic as the medium for high-risk cases where chances of survival are low and such treatment may avoid fatality due to the COVID-19.
\end{abstract}

INDEX TERMS COVID-19, Ultraviolet (UV), far-UVC, fiber optics, selective treatment

\section{INTRODUCTION}

COVID-19 is the respiratory disease which occurs due to the severe acute respiratory syndrome typically referred to as SARS-CoV-2 virus [1]. Around 100 years after the pandemic influenza of year 1918, COVID-19 has inevitably transformed 2020 into an era of pandemic and global lockdowns [2][3]. The global economies are struck with a downfall admist the unavailibity of task force, the homo sapiens. The global health care system has exhausted. Though, frontline warriors are around the clock exploring and experimenting for the cure, vaccination and diminishing of the virus. As the spread of COVID-19, worldwide without discrimination of race, cast, color or creed, the sought solution againt COVID19 need not only to be erradiate the disease but also, needs to be economical. Another important factor is the accessibility and reachibility of the solution, specifically in the developing countries, in order to hault the assomptatic natured virus which owns enormous multiplicity feature and can thus, always impact globally.

Inorder to combat the multiplicity of COVID-19, ultraviolet (UV) devices are brought into use for decontamination of diverse surfaces such as hospital rooms, masks and even, buses. UV light has been demonstrated to be capable of destroying viruses, bacteria and fungi in hundreds of laboratory studies [4].With respect to wavelength, UV radiation can be categorized as:
UVA having range of 400-320 $\mathrm{nm}$, UVB having range of $320-290 \mathrm{~nm}$ and UVC having range of 290-200 nm). The UVA type of radiations leads to light brown tan after a small-time span; the consequent darkening occurs because of melanin, that gathers in the skin. The UVB radiation causes extreme sunburn, related with intensified erythema and edema, ache, and formation of blister in lesser than a day of exposure. UVC is referred to as a germicidal radiation which due to its effectiveness in killing microorganisms owns sterilization and biocidal features, as well [11].

Infact, UV blood irradiation (UBI) was widely used during 1940s to 1950 s for treatment of various diseases such as septicemia, pneumonia, tuberculosis, arthritis, asthma and even poliomyelitis as figure 1 gives a peek into history[5]. Particulary, Far-UVC light, having range of $207-222 \mathrm{~nm}$, has been investigated for efficiently inactivating bacteria without being toxic to bared mammalian skin. Far-UVC has strong absorbance in biological materials because of which it cannot pass through even the external human skin layers or eye; although, far-UVC can pass through and inactivate bacteria as well as viruses as their size is of micrometer or lesser.

Hence, this work motivates far-UVC as an accessible and cost-effective treatment for COVID-19 in humans. The work emphasizes that far UV-C lights and treating selective regions in COVID-19 patients through 


\begin{tabular}{|ll|}
\hline $\begin{array}{l}\text { Year } \\
\text { Bonnet }\end{array}$ & Form of UV \\
\hline $\begin{array}{l}\mathbf{1 8 5 5} \\
\text { Rikli }\end{array}$ & Sunlight for tuberculosis arthritis \\
\hline $\begin{array}{l}\mathbf{1 8 7 7} \\
\text { Downes and Blunt }\end{array}$ & Thermal station for helio-therapy \\
\hline $\begin{array}{l}\mathbf{1 9 0 4} \\
\text { Niels Finsen }\end{array}$ & Nobel Prize for UV treatments of skin conditions \\
\hline $\mathbf{1 9 2 0 s , \text { Walter }}$ & High cure rate for erysipelas using Uvlight \\
\hline $\mathbf{1 9 2 8}$ & UBI for Septic abortion intricated by hemolytic streptococcus septicemia \\
\hline Hancock and Knott & UBI for hemolytic streptococcal septicemia. \\
\hline George P Miley & $\begin{array}{l}\text { Use of process for treating thrombophlebitis, botulism, poliomyelitis, non-healing wounds, staphylococcal } \\
\text { septicemia, peritonitis and asthma }\end{array}$ \\
\hline $\mathbf{1 9 4 0}$ & $\begin{array}{l}\text { Infectious arthritis, septic abortion, uveitis, furunculosis, osteoarthritis, tuberculosis glands, chronic blepharitis, } \\
\text { mastoiditis, chronic paranasal sinusitis, acne vulgaris, and secondary anemia }\end{array}$ \\
\hline
\end{tabular}

Table 1. UV treatment in History

fiber optics based far UV-C is a practical choice for patients where the survival chances are already low, and fatality because of COVID-19 could possibly be avoided using far UV-C. The major contributions of the work are as follows:

- The works focusing on far-UVC for healthcare are briefed.

- The significance and potential of far UV C for the decontamination of COVID-19 in humans is discussed.

- Relevant imperative research questions are highlighted.

The rest of this paper is organized as follow. Section II highlights the works which are target UV for healthcare. In Section III, the potential of Far-UVC for humans are discussed and research questions are raised by Section IV. Section V presents the conclusion of the paper.

\section{RELATED WORKS}

As an ancestral cure, the research of [5] emphasized that effectiveness UV treatments has been forgotten with passage of time. The work is motivated by emergence of antibiotic-resistant-bacteria and promotes UltraViolet Blood Irradiation (UBI) as an immune-modulating therapy. It also highlighted major works which had previously utilized UV for health-related issues.

However, the current literature reflects a rise of explorations on a particular range of UV referred to as the far-UVC. The study of [6] establishes that far-UVC, 224nm UV radiation, $\mathrm{UV}_{224}$, supplied from laser over an optical diffuser happens to be viable retort for decontamination of susceptible area, for example skin penetrating medical devices, catheters or drivelines. The novel study showed that far-UVC irradiation supplied by usage of fiber optics is favorable technology to prevent infections instigated through contamination by bacteria. A good example of the technology is to use laser for conveyance of far-UVC light by means of an optical fiber and radiated from diffuser is remarkable. In [7], extremely low doses of far-UVC light were competently used to deactivate airborne viruses conveyed by aerosols. By use of 222-nm UV radiation $\left(\mathrm{UV}_{222}\right)$, [8] investigated that how $\mathrm{UV}_{222}$ eradicates cultivating bacteria and spores, and the factors which are imperative for allowing cells and spores to defy the effects of this radiation. $\mathrm{UV}_{222}$ is theoretically a safer alternate to UV254 which is frequently utilized for surface sanitization. For in vitro investigation of [9], laser generated far UVC light is carried using an optical diffuser for deactivating Methicillin-resistant Staphylococcus aureus (MRSA) on surface by means of far-UVC light from a laser conveyed using an optical diffuser. Analysis reflected that the exposure of far-UVC is ample to inactivate MRSA with a dosage equivalent to the one needed using a typical germicidal lamp. Results indicate that far-UVC competently deactivates airborne aerosolized viruses, with an extremely small dose of $2 \mathrm{~mJ} / \mathrm{cm} 2$ of $222-\mathrm{nm}$ light inactivating $>95 \%$ of aerosolized $\mathrm{H} 1 \mathrm{~N} 1$ influenza virus. The safe use of UV-based sterilization was developed in [10] where a far-UVC having single wavelength of 207, $\mathrm{UV}_{207}$, was used for exterminating bacteria, without impairing the cells or tissues in human.

In other works, 254-nm UV radiation, $\mathrm{UVC}_{254}$, was probed exposure-period dependently for the ultrastructural effects of on cells of lung in mole rats. Similarly, $\mathrm{UVC}_{254}$ was used for pre-clinical study to demonstrate the capability of lightbased therapies - UVC and photodynamic therapy (PDT) during Ex vivo lung perfusion (EVLP) to deactivate hepatitis $\mathrm{C}$ virus (HCV) in donor lungs. In a stepwise application of this technology to clinical transplantation, a clinical trial was 
designed to assess effects of UVC treatment using NAT+ HCV donor lungs trailed by transplantation.

\section{POTENTIAL OF FAR-UVC IN COVID-19 FOR HUMANS}

The potential of far-UVC in humans against COVID-19 is three-fold: 1) far-UVC lights for public spaces 2) FarUVC for selective treatment in high risk paitents 3) FarUVC for diverse range of sterilization purposes. We will discuss the first two here:

\section{A. Far-UVC lights for public space}

Antimicrobial aptitude of UVC ultraviolet light is widely accepted in history. However, owing to the health hazard related side effect, public spaces don't employ them as mean to kill viral infections. However, the work of [7] effectively shows that an extremely squat dosage of 2 $\mathrm{mJ} / \mathrm{cm}^{2}$ of $222-\mathrm{nm}$ light deactivates greater than $95 \%$ of airborne H1N1 virus. The usage of overhead low-level far-UVC light in public spaces might present a nontoxic and effectual technique for controlling the spread of airborne-mediated microbial disease. Utilization of lowlevel far-UVC fixtures provision desired antimicrobial advantages with no human health related concerns which existed with conventional germicidal lamp UVGI associated human health concerns of conventional germicidal lamp. Hence, far-UVC lights are an economical candidate for the global pandemic of COVID19. As COVID-19 spreads with asymptomatic feature, introduction of far-UVC lights is immensely important for a safer world. Investigations and guidelines are needed for placement of far-UVC lights in public settings which can be timely handled. Introduction of such an infrastructure which involves strategic placement of far-UVC lights will be effective for not only COVID-19, but all multi-drugresistant variants of bacteria. Public locations for example, schools, hospitals, offices, schools, doctors' airplanes and airports might be considered here.

\section{B. Far-UVC for selective Treatments in Human}

UV radiation, implicitly, influence toxic effects on living beings. In [11], mole rats were exposed to $\mathrm{UVC}_{254}$. In the tissue of rat lung fore-most detectable modifications existed after 14 days of exposure to UVC radiation. As already highlighted, far-UVC light can effectively deactivates bacteria with no damage to exposed mammalian skin. The biophysical reason is that the range of far-UVC light is not ample range to pass the the external layer (stratum corneum) of human skin. Also, far $\mathrm{UV}-\mathrm{C}$ cannot penetrate the external tear layer of eye, because to its strong absorbance in biological materials. Though, as viruses as well as bacterias are typically have dimension of micron or even lesser size.
In many cases, the source of far-UVC light for example excimer lamp would not be ample for decontamination as implanted lines function as protecting for most of wound region. Moreover, the far-UVC light cannot pass through the skin to decontaminate within the wound. A possible answer to these circumstances is to direct light around and inside complicated geometries by use of fiber optics. The fiber optics, which can be paired with a far-UVC area laser as a source of light, could be maneuvered to send light to various regions around proneto-infection area. Coupling the fiber optic with a diffusing element which is able to emit the light radially from the fiber would permit for application over a greater target area in the percutaneous areas which would not haven possible to expose otherwise. The development of such methodology for decreasing infection at skin entry are while being most probably safe for human exposure is important for the fight against drug-resistant bacteria, specially in hospital settings [9].

Current trends in far-UVC laser technology development have reflected potential for shrinking the size as well laser module's expense for suitability of large-scale adoption. Consider the example from Europe, Sharp Laboratories have developed condensed UVC lasers which are as small as the AA battery; although, the laser module utilized for the work was $10 \mathrm{~cm} \times 10 \mathrm{~cm} \times$ $70 \mathrm{~cm}$ having around $4 \mathrm{~kg}$. Compact laser modules could empower the creation of decontamination systems using far-UVC technology which are effortlessly portable and hence capable of being applied to diverse range of applications [9].

In regards with COVID-19, far-UVC lasers for decontamination could be advantageous from enhanced light diffusing technology which is well suited for the application. Owing to complexity in functioning with wavelengths of far-UVC, greatly because of effects of solarization which decrease transmittance, commercially available optical parts are restricted. New diffusers that are greatly robust and highly compatible to unique biological locations which they will be utilized for will also enhance technology. Thus, the potential of far-UVC as safe, and effective technology that diminishes will for an antimicrobial tool which works.

In-vivomouse skin results consistent with prior investigations in a 3-D human skin tissue in vitro model [8] which also presented imperative skin damage after typical $U_{254}$, but none after $U_{207}$. The outcome readily interpretable with respect to limited range of $207 \mathrm{~nm} \mathrm{UV}$ light which cannot pass through the stratum corneum surface layer of mouse or human skin; on the contrary, broad spectrum UVC from a $254 \mathrm{~nm}$ germicidal lamp can swiftly pass through the and dermis and epidermis. Prior works also recommended that $\mathrm{UV}_{207}$ light and $\mathrm{UV}_{254}$ from a germicidal lamp are approximately equitoxic to MRSA. 
Moreover, recent investigations have reflected on an open wound with no protective layer of stratum corneum that its irradiation of an open wound with UVC light of $222 \mathrm{~nm}$ did not stimulate DNA damage for the target cells. However, the bactericidal was still under control.

Therefore, owing to its immense potential, a technique for fiber optics based far-UVC must be investigated for selective treatment of COVID-19. By using fiber optics as the medium, most suspected region such as organs in terms of locality of COVID-19, can be selectively treated with exposure to far-UVC.

\section{RESEARCH QUESTIONS}

The major research questions whose answers need to seek in regards with use of far-UVC on humans in battle against COVID-19 are stated as follows:

1. What should the effective exposure time of farUVC?

2. What frequency of far-UVC would be good enough against COVID-19?

3. What will be the associated side effects in humans in response to exposure with far-UVC?

4. Which fiber optic will be most suitable for the procedure?

5. How can this strategy of far-UVC be adopted for public places?

\section{CONCLUSION}

Far-UVC has immense potential as an economical way for treating COVID-19. High risk cases of COVID-19 where the chances of survival are already low, far-UVC can be employed as the last resort providing tradeoff between the fatality and survival. For high risk cases, exposure to farUVC lights and treatment of selective regions using fiber optics induced far-UVC must be investigated to combat COVID-19 and probable pandemics for a safer tomorrow.

\section{REFERENCES}

[1] Fisher, Dale, and David Heymann. "Q\&A: The novel coronavirus outbreak causing COVID-19." BMC medicine 18.1 (2020): 1-3.

[2] Morens, David M., and Jeffery K. Taubenberger. "The mother of all pandemics is 100 years old (and going strong)!." American journal of public health 108.11 (2018): 1449-1454.

[3] Remuzzi, Andrea, and Giuseppe Remuzzi. "COVID-19 and Italy: what next?." The Lancet (2020).

[4] Armellino, Donna, et al. "Assessment of focused multivector ultraviolet disinfection with shadowless delivery using 5-point multisided sampling of patient care equipment without manual-chemical disinfection." American journal of infection control 47.4 (2019): 409-414.
[5] Hamblin, Michael R. "Ultraviolet irradiation of blood:" the cure that time forgot"?." Ultraviolet Light in Human Health, Diseases and Environment. Springer, Cham, 2017. 295-309.

[6] Welch, David, et al. "Effect of far ultraviolet light emitted from an optical diffuser on methicillin-resistant Staphylococcus aureus in vitro." PloS one 13.8 (2018).

[7] Welch, David, et al. "Far-UVC light: A new tool to control the spread of airborne-mediated microbial diseases." Scientific Reports 8.1 (2018): 1-7.

[8] Taylor, Willie, et al. "DNA Damage Kills Bacterial Spores and Cells Exposed to 222-Nanometer UV Radiation." Applied and Environmental Microbiology 86.8 (2020).

[9] Welch, David, et al. "Far-UVC light applications: sterilization of MRSA on a surface and inactivation of aerosolized influenza virus." Light-Based Diagnosis and Treatment of Infectious Diseases. Vol. 10479. International Society for Optics and Photonics, 2018.

[10] Buonanno, Manuela, et al. "207-nm UV light-a promising tool for safe low-cost reduction of surgical site infections. I: in vitro studies." PloS one 8.10 (2013).

[11] Türker, Hüseyin. "Histological and ultrastructural analyses of mole rats lung cells exposed to ultraviolet radiation." Journal of Radiation Research and Applied Sciences 7.4 (2014): 560-567.

[12] Cypel, M., et al. "A clinical trial evaluating the effects of ultra-violet $C$ treatment (UVC) during ex vivo lung perfusion (EVLP) as a method of inactivating hepatitis $\mathrm{C}$ infection in donor lungs." The Journal of Heart and Lung Transplantation 38.4 (2019): S53-S54. 